duration of disease at 1st assessment was 7.0 years (IQR 5.5-8.8). 24 patients were transited successfully. Only $60 \%$ of our youths felt that transition preparation is important and had confidence to be prepared for/ change to adult healthcare system (AHCS). Among the less confident group (scores <7), MKHU, MM, AM and HT domains scored significantly less than those from confident youths $(p<0.001-0.021)$, but only transition importance MKHU scores were $<7$. Disease duration at first TRAT assessment and diagnoses did not predict confidence level. With further targeted education and counselling (median 8.3 months (IQR 5.7-9.6)), confidence and MKHU was significantly improved $(p=0.001)$.

Conclusion: TRAT enables our PRTP team to learn and understand our local youths' perception and readiness of adult transition process. More than one-half of them did not aware of the importance of the transition process and had less confidence to do so. With further targeted counselling and preparation, all improved their perception and MKHU significantly. TRAT was able to guide individualize counselling to our cohort youths who are ready to be transited.

Acknowledgement: American College of Rheumatology

Disclosure of Interests: Sook Fun Hoh: None declared, Xiaocong Gao: None declared, Yun Xin Book: None declared, Lena Das: None declared, Thaschawee Arkachaisri Speakers bureau: Abbvie Pte, Ltd

DOI: 10.1136/annrheumdis-2019-eular.5169

\section{THU0697-HPR CONTENT OF AND RHEUMATOLOGISTS' AND NURSES OPINIONS ON A NURSE-LED TELEPHONE HELPLINE}

Mehmet Elmacioglu $^{1,2}$, Natalia Westerman ${ }^{3}$, Debby Vosse ${ }^{1}$, Annelies Boonen ${ }^{1}$, Yvonne van Eijk-Hustings ${ }^{1} .{ }^{1}$ Maastricht University Medical Centre, Maastricht, Netherlands; ${ }^{1}$ Maastricht University Medical Centre, Maastricht, Netherlands; ${ }^{3}$ Maastricht University, Maastricht, Netherlands

Background: To improve timely response to patients' questions and to support that care is provided by the appropriate professional, a nurse-led rheumatology telephone helpline was introduced in May 2015. Different organisational approaches have been tried out to ensure an effective as well as efficient service. Currently a voicemail system is used. A selection menu allows patients to select whether he/she needs 1) advice regarding disease complaints, 2) drug prescriptions, 3) (change in) appointment at the clinic, 4) laboratory forms.

Although the menu differentiates between requests, organising timely responses by the appropriate professional remains a challenge. Clear and joint definitions by the rheumatologists and nurses on what questions to what extent should be addressed by whom, are necessary.

Objectives: To facilitate further improvement of the helpline we explored 1) number and content of questions that were registered; 2) experiences of rheumatologists and nurses with the helpline; 3) opinions of rheumatologists and nurses on which questions should be answered via the helpline.

Methods: A file research explored the number of questions and reasons for contacting the helpline. Data were analysed descriptively. In semistructured interviews among all rheumatologists $(n=8)$ and nurses involved in manning the helpline service $(n=6)$, experiences and opinions were explored. System Engineering Initiative Patient Safety (SEIPS) model, that focuses on system design and it's interaction with processes, and outcomes for patients, employees and organisation [1], was used to structure thematic coding of the transcripts.

Results: In 37 months, the helpline registered 3389 calls (see Table 1) A majority of the questions was about disease complaints (28.6\%) or other related problems such as pain management, and exercise (27.8\%). The proportion of requests for prescriptions was $21.8 \%$.

The interviews revealed that both rheumatologists and nurses considered the helpline valuable in providing easy access to care for patients with disease related questions. This should be the primary aim. The rheumatologists also valued their reduced workload, which occurred most if the knowledge of the nurse was sufficient to take care of the responses independently. Moreover, they valued if requests, such as prescriptions, were clustered by the nurses. The nurses experienced an increased workload. Sufficient knowledge on and training in rheumatology, and being familiar with the patient wee considered pivotal in providing appropriate and for the patient acceptable care. As nurses cannot precribe medication, the requests for prescriptions were considered inappropriate. Availability of protocols and guidelines were considered prerequisites by all for responding to patients' requests appropriately.

Conclusion: The helpline is used frequently and the selection menu has helped to gain insight in why the helpline is used by patients. There is consensus on the primary aim of the helpline and on prerequisites for nurse-led responses to patients' disease related questions. Rheumatologists and nurses have different opinions on some issues, such as the organisation of prescriptions. Our study findings can help in jointly improving consensus on the content of nursing care within the helpline.

\section{REFERENCES:}

[1] Carayon, P., et al. (2006). Work system design for patient safety: The SEIPS model. Qual Saf Health Care, 15(SUPPL. 1), 50-58

Abstract THU0697HPR Table 1. Reasons for contacting the helpline

\begin{tabular}{lc}
\hline & Calls May 2015-July 2018 \\
\hline Reasons & $\mathbf{N}(\%)$ \\
Disease complaints & $970(28.6)$ \\
Medication prescriptions & $739(21.8)$ \\
Medication side-effects & $353(10.4)$ \\
Appointments & $184(5.4)$ \\
Lab forms & $126(3.7)$ \\
Other & Total \\
\hline
\end{tabular}

*Other: a variety of requests e.g. advice for pain management, exercise, work participation, referrals for devices.

Disclosure of Interests: Mehmet Elmacioglu: None declared, Natalia Westerman: None declared, Debby Vosse: None declared, Annelies Boonen: None declared, Yvonne van Eijk-Hustings Grant/research support from: UCB, Speakers bureau: Cellgene

DOI: 10.1136/annrheumdis-2019-eular.2624

\section{THU0698-HPR MORE EFFICIENT DMOAD TRIALS THROUGH INNOVATIVE SCREENING STRATEGIES}

Thibault Helleputte $^{1}$, Anne-Christine Hick ${ }^{2,3}$, Yves Henrotin ${ }^{2,3} .{ }^{1}$ DNAlytics, Louvainla-Neuve, Belgium; ${ }^{2}$ Artialis SA, Luik, Belgium; ${ }^{3} \mathrm{CHU}$ Liège, UROC, Liège, Belgium

Background: In randomized Clinical Trials (RCT), the number of patients to recruit for assessing effectiveness of disease-modifying $O A$ drug (DMOAD) depends on the proportion of progressors in the population. The higher this proportion, the smaller the cohort to be recruited. In the general $O A$ population, this proportion is quite low (10-30\%) [1] requiring large cohorts, hence long and expensive trials.

Objectives: We study how predictive modeling based on early cartilage degradation biomarkers can be used to screen patients, make individual predictions about the likelihood of future progression, and recruit on this basis a progressor-enriched cohort. By doing so, DMOAD RCTs could achieve same or better success rates based on smaller cohorts and lower budgets, even taking into account the extra cost of this innovative screening.

Methods: Two biomarkers (Coll2-1 \& Coll2-1-NO2, Artialis, Belgium) have been measured at baseline on 182 OA patients from the placebo arm of a previous RCT. For this cohort, progression at $30 \mathrm{M}$ is defined as in [2]. We developed two predictive models of the progression of the patients over 30 months through regularized logistic regressions: one based on Coll2-1 only, the other on Coll2-1 and Coll2-1-NO2. Based on these models, we design a strategy to produce cohorts of patients with a higher rate of progressors than the natural proportion. To do so, we make hypotheses on the expected drug effect (from $30 \%$ to $50 \%$ ), on the costs generated by a trial designed according to a regular strategy (i.e. not using baseline markers for patients inclusion/exclusion), and on the costs generated by this innovative screening strategy (a.o. nb patients screened, enrolled, costs of visits, imaging, biomarkers when applicable) Based on these hypotheses, we optimize sensitivity/specificity trade-offs of each model, so as to reduce the sample size required to prove the expected effect.

Results: When we apply this strategy to realistic scenarios, both models produce substantial cost reductions (up to 30\%) even considering the extra cost of the biomarker-based screening of a higher number of candidate participants. They also lead to cohort size reductions: almost $40 \%$ less patients needed. As an example, we considered a natural progressor rate of $20 \%$, an actual effect of $50 \%$ for the DMOAD to be tested, and a study consistsuing in a screening visit, 4 follow-up visits, 2 x-rays, 2 MRI, and a final visit. Hypotheses have been made about costs of each of these items, according to Belgian practice. In the standard case, all screened patients satisfying inclusion criteria are recruited. In the modified version, only patients for which inclusion criteria are met and the tuned biomarker-based model turns positive are enrolled. In the standard version of such a study, 554 patients have to be recruited, with a cost of $1,767,260 €$. Through the optimized strategy, 1190 patients are screened, 